\title{
Integrated Care Systems and the Aortovascular Hub
}

\author{
Mark Field ${ }^{1,2}$ Manoj Kuduvalli ${ }^{1}$ Francesco Torella ${ }^{1}$ Victoria McKay ${ }^{1}$ Afshin Khalatbari ${ }^{1}$ \\ Gregory Y. H. Lip ${ }^{1,2, * \mathbb{D}}$
}

${ }^{1}$ Aortovascular Hub, Liverpool Heart and Chest Hospital, Liverpool,
United Kingdom
${ }^{2}$ Liverpool Centre for Cardiovascular Sciences, Liverpool Heart and
Chest Hospital, Liverpool, United Kingdom

Address for correspondence Mark Field, Aortovascular Hub, Liverpool Heart and Chest Hospital, Liverpool L14 3PE, United Kingdom

(e-mail: mark.field@Ihch.nhs.uk).

Thromb Haemost 2022;122:177-180.

\section{Introduction}

Aortovascular disease, medicine, and surgery are relatively new terms that have appeared in the literature and in the designations and names of individuals, symposia, and institutions over the past 5 years, predominantly in the United Kingdom where cardiac surgery and vascular surgery are separate specialties and vascular medicine is not commonplace. We have recently attempted to define the term aortovascular medicine ${ }^{1}$ as a coordinated multispecialty approach to a unique subgroup of patients with vascular disease. The general approach represents an example of an integrated care system (ICS) which forms part of the NHS (National Health Service) Long Term Plan with a multidisciplinary, multiagency approach to lifelong care of patients in local communities (https://www.kingsfund.org.uk/publications/ integrated-care-systems-explained). This "clinical concept" article will attempt to explore the multidisciplinary networks that define this approach and explain the novel concept of the Aortovascular Hub as an ICS (- Fig. 1). While not exclusively the case, much of the pathology addressed by aortovascular medicine and surgery relates to thoracic arterial disease and in particular syndromic phenotypes such as Marfan syndrome, Ehlers-Danlos syndrome, Loeys-Dietz syndrome, Turner syndrome, bicuspid aortic valve syndrome, and unspecified familial and nonfamilial gene-based processes. There is increasing recognition that patients with developmental anomalies, particularly those with dysplastic aortic arch and aneurysmal epiaortic vessels including aortic coarctation, are included in this group. The term "aortovascular" was first used to include the continuum of peripheral "nonaortic," vascular manifestations of aortopathy, and this still remains the essence of aortovascular disease, medicine,

\footnotetext{
* The review process for this paper was fully handled by Christian Weber, Editor in Chief.
}

received

July 20, 2021

accepted

August 17, 2021

published online

August 18, 2021 and surgery. These pathologies are somewhat distinct from acquired degenerative arterial aneurysms and occlusive disease that accompany abdominal aortic pathology, peripheral carotid, and iliofemoral disease-the core practice of vascular surgeons. While not excluding degenerative disease, the nuances of the thoracic aortopathies and the requirement for broad multidisciplinary involvement in patient investigation, diagnosis and management, and broader social integration are the focus that in turn define aortovascular medicine. Operations on the thoracic aorta involving the aortic root and total arch are traditionally the realm of cardiac surgeons, and those involving aortic endovascular approaches are traditionally the realm of vascular surgeons. However, aortovascular surgery blurs some of these boundaries and partly describes collaborative, integrated, and holistic working on open approaches to the chest and neck as well as hybrid open/endovascular approaches. Such an integrated approach follows developments in other disease areas for a more holistic approach to improving patient care pathways, for example, in patients with atrial fibrillation. ${ }^{2}$ Such integrated care approaches have been associated with a significant reduction in adverse outcomes. ${ }^{3,4}$ Aortovascular medicine and surgery in relation to vascular health of the population therefore requires input from a broad range of specialists and institutions throughout a patient's life as well as integration with allied health care providers. This represents essence of the "Aortovascular Hub" concept.

\section{Aortovascular Hub: Disease Etiology and Pathology}

By far the commonest vascular arterial disease is atherosclerotic occlusion with age, blood pressure, smoking, diet, diabetes as well as socioeconomic and behavioral factors as major risk factors. The majority of this disease is extrathoracic. A significant proportion of thoracic aortic disease is more

$\begin{array}{ll}\text { (C) 2021. Thieme. All rights reserved. } & \text { DOI https://doi.org/ } \\ \text { Georg Thieme Verlag KG, } & 10.1055 / \mathrm{a}-1591-8033 .\end{array}$ Georg Thieme Verlag KG,

Rüdigerstraße 14,

70469 Stuttgart, Germany
ISSN $0340-6245$. 


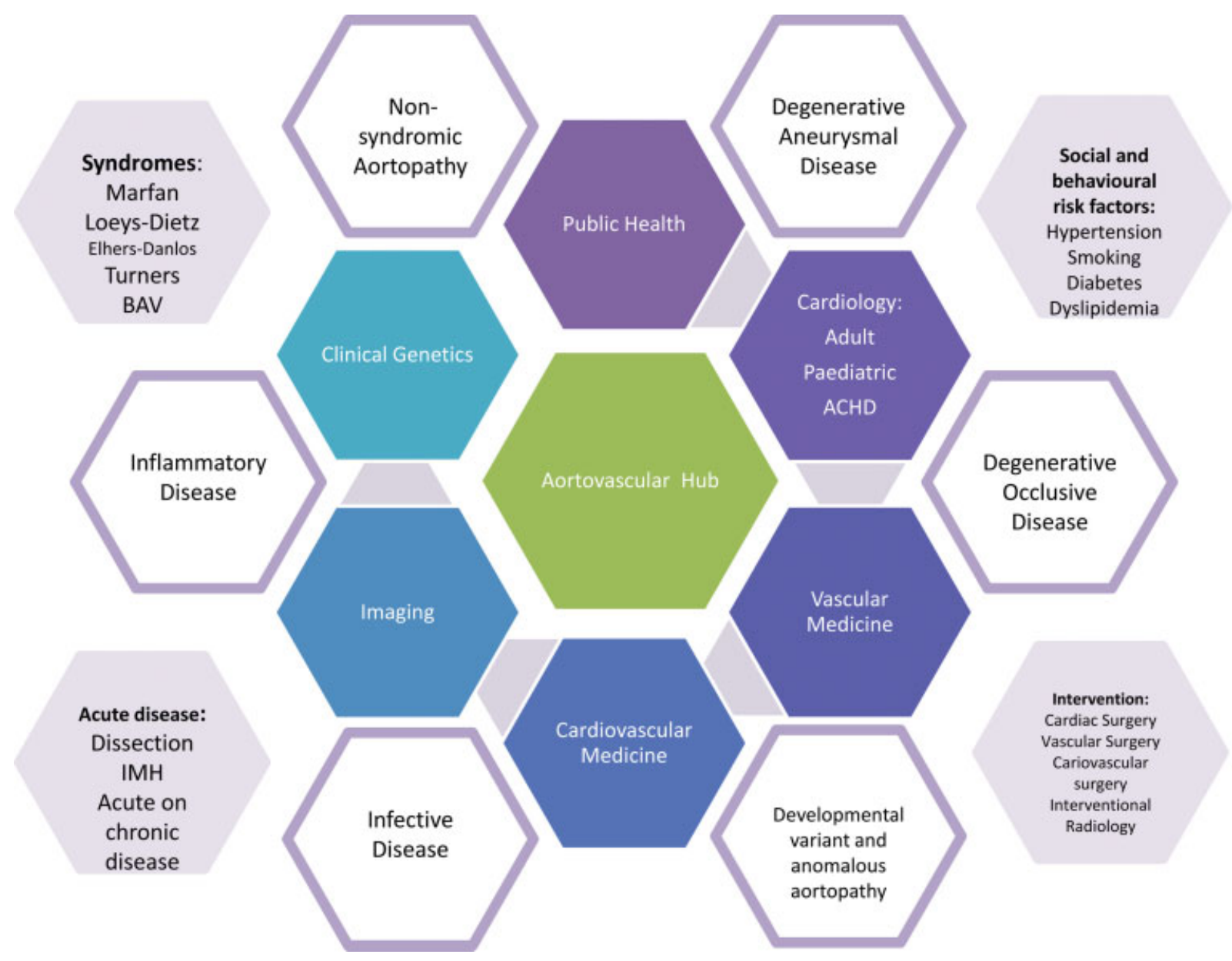

Fig. 1 The Aortovascular Hub.

complex with genetic and or developmental etiology. The socalled "aortopathies" are typically syndromic or nonsyndromic, the latter being sporadic or familial. The syndromic aortopathies including Marfan syndrome, Ehlers-Danlos, Loeys-Dietz, Turners, and bicuspid aortic valve syndromes often have vascular manifestations extending beyond the aorta as well as extracardiovascular pathology requiring multidisciplinary care. The distinct patterns of arteriopathy or aortovascular anatomy within these aortopathies set them apart from degenerative disease and make them uniquely suitable to an aortovascular multidisciplinary approach. While aortic root aneurysms dominate the vascular pathology of syndromic disease and nonsyndromic familial/sporadic disease, developmental variants and anomalies are an important etiology accounting for aortic arch pathologies. Rare developmental pathologies such as aberrant subclavian arteries, right-sided arch, and vascular rings are found as sporadic disease or part of syndromes such as CHARGE syndrome. Indeed, dysplastic aneurysmal distal aortic arch and left subclavian artery represent a pathology uniquely suited to an aortovascular approach (-Fig. 2). A significant number of these patients also present with acute aortic syndromes including intramural hematoma, dissection, and rupture.

\section{Aortovascular Hub: Medicine}

Several countries already have specialties which manage many of the arterial pathologies described, including vascular medicine, cardiovascular medicine, angiology, and cardiology as well as to some extent stroke medicine and renal medicine. However, aortovascular medicine focuses primarily on clinical genetics of aortopathy, vascular imaging, and quite often the myriad of extravascular anomalies of syndromic disease. This requires additional skills to that required to manage traditional degenerative, atherosclerotic disease processes that affect the majority of the population. With the advent of a large number of newly identified genetic variants, some of uncertain significances, some with aggressive phenotype, the natural history, and associated risks of acute aortic syndrome, are not as predictable as degenerative disease. The input of specialized clinical genetics to the aortopathy MDT (multidisciplinary team) and patient-specific risk-based management becomes mandatory. The interplay between clinical genetics, imaging cardiology, and surgeon becomes the central triumvirate of decision making. However, additional subspecialty input into the MDT is often required by those with an interest in radiology, vasculitis, 


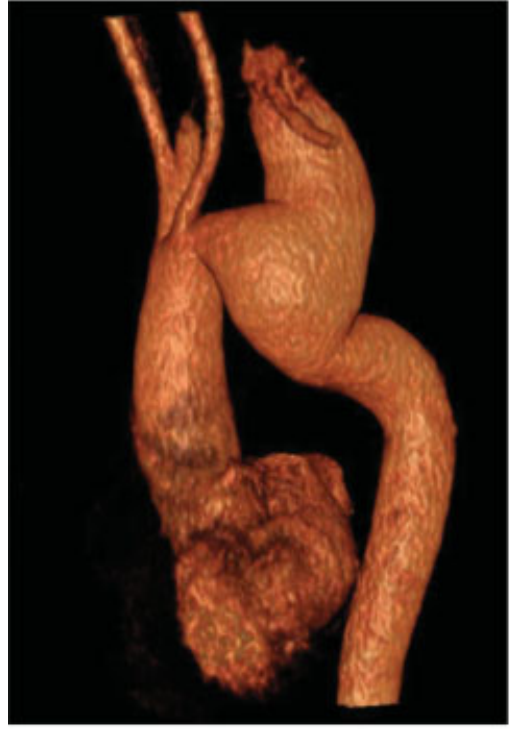

A

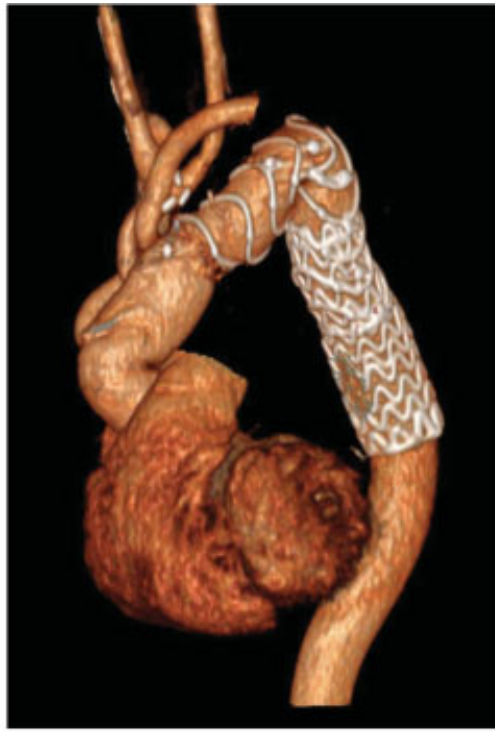

B

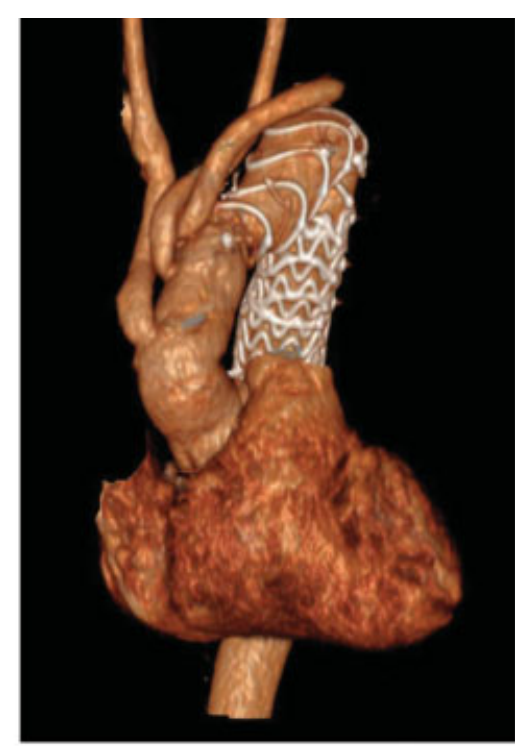

C

Fig. 2 A 32-year-old man presented with a bicuspid aortic valve and a developmental aneurysmal dysplastic left subclavian artery (LSA) (A). The patient required an aortic valve replacement, total aortic arch, and frozen elephant trunk with rerouting of LSA from supraclavicular course into the chest, with a TEVAR extension (B, C), skills not found in an individual within the UK system. Collaborative working allowed this man to have safe intervention via a single surgical incision.

and resistant complex hypertension as well as adult congenital heart disease and perioperative medicine in the form of anesthesia and intensive care.

\section{Aortovascular Hub: Surgery}

Aortic surgery performed by cardiac surgeons has become very nuanced and sophisticated with Bentall root replacements evolving into valve sparing root replacements, Ross operations, and total aortic arch operations under deep hypothermic circulatory arrest. Open descending aortic and thoracoabdominal aortic surgery has evolved to safer intervention but typically in the realm of cardiac surgery with skills in cardiopulmonary bypass. Aortic surgery performed by vascular surgery has also "migrated" more proximally with the development of complex endovascular intervention extending from thoracoabdominal treatment to aortic arch and even to the aortic root. The development of the composite surgical branched arch graft and TEVAR (thoracic endovascular aneurysm repair or frozen elephant trunk) metaphorically represents the increasingly blurred boundary between these two specialties within the United Kingdom that epitomizes the aortovascular approach to surgery. - Fig. 2 represents an example of the collaborative aortovascular approach to a challenging developmental anomaly of the distal aortic arch. Aortovascular surgery should not be based solely upon the specifics of aortopathy and anatomy. Degenerative, infective, and inflammatory diseases are often conducive to such hybrid interventions and collaborative working. Additionally, acute aortic syndromes may be best treated under the aortovascular model. These are often driven by a desire to reduce the extent of open surgery, the need for cardiopulmonary bypass, and to avoid deep hypothermic circulatory arrest.

\section{Aortovascular Hub: Experience in Liverpool}

Aortovascular medicine has evolved within Liverpool with the acknowledgment that the diagnosis, investigation, and management of complex patients are no longer within the scope of practice of surgeons alone.

Aortovascular medicine has evolved as a multidisciplinary, multi-institution team with the skills to manage this cohort of patients, together forming a virtual hub (-Fig. $\mathbf{1})$. Several developments delivering this service include an inherited aortopathy clinic as well as MDTs for aortopathy, aortic aneurysms, and adult congenital heart disease, as well as integrated clinical genetics services. We do not see aortovascular medicine developing as a specialty in its own right but rather a novel collective holistic "integrated care" effort. One of the key functions of the aortovascular hub is to provide patient-specific, lifelong, multidisciplinary care of cardiovascular and extracardiac diseases, especially for syndromic and adult congenital patients. A key element of the approach is the development of integrated care pathways which direct patients through lifelong multidisciplinary care, with multiple entry points dependent on patient cohort, additionally managing social and behavioral aspects.

\section{Conclusion}

Integrated care pathways, and more recently as part of the NHS Long Term Plan, ICSs, represent the direction of travel within the NHS. The creation of an aortovascular hub within the Cheshire and Merseyside Health and Care Partnership represents an important step in acknowledging the multidisciplinary, multiagency approach to a group of patients that transcends traditional medically defined specialties, functioning in silos and in a competitive approach. 
Aortovascular patients, with multisystem disorders requiring "joined up" coordinated care over a lifetime, are uniquely positioned to benefit from this approach. The challenge is for clinicians and organizations to look beyond isolated priorities and focus on system-wide objectives to improve the outcomes for the communities they serve, whether these be vertical or horizontal provider collaboratives. The concept of the "Aortovascular Hub," as an ICS, is likely to be one of many emerging initiatives that change our thoughts around traditional models for delivery of excellent clinical care.

Conflict of Interest

None declared.

Acknowledgments

We would like to acknowledge the wider team including: Una Ahern, Steve Akrigg, Sarah, O'Leary, Ahmed Othman, Omar Nawaytou, Debbie Harrington, Caroline McCann, Janice Harper, John Brennan, Johnathan Smout, Simon
Neequaye, Rob Fisher, Rao Vallabhaneni, Richard McWilliams, Johnathan Kendall, as well as a series of cardiac and vascular aortic fellows.

\section{References}

1 Field ML, Kuduvalli M, Torella F, Lip GY. Aortovascular medicine: what is it? J R Soc Med 2021. Doi: 10.1177/01410768211013436

2 Lip GYH. The ABC pathway: an integrated approach to improve AF management. Nat Rev Cardiol 2017;14(11): 627-628

3 Romiti GF, Pastori D, Rivera-Caravaca JM, et al. Adherence to the 'Atrial Fibrillation Better Care' pathway in patients with atrial fibrillation: impact on clinical outcomes-a systematic review and meta-analysis of 285,000 patients. Thromb Haemost 2021. Doi: 10.1055/a-1515-9630

4 Yoon M, Yang PS, Jang E, et al. Improved population-based clinical outcomes of patients with atrial fibrillation by compliance with the simple $\mathrm{ABC}$ (Atrial Fibrillation Better Care) pathway for integrated care management: a nationwide cohort study. Thromb Haemost 2019;119(10):1695-1703 